\title{
Level of serum undercarboxylated osteocalcin correlates with bone quality assessed by calcaneal quantitative ultrasound sonometry in young Japanese females
}

\author{
YOSHIO SUZUKI $^{1}$, ASAKO MARUYAMA-NAGAO ${ }^{2}$, KEISHOKU SAKURABA ${ }^{1}$ and SACHIO KAWAI ${ }^{1}$ \\ ${ }^{1}$ Graduate School of Health and Sports Science; ${ }^{2}$ School of Health and Sports Science, \\ Juntendo University, Inzai, Chiba 270-1695, Japan
}

Received January 4, 2016; Accepted January 3, 2017

DOI: $10.3892 /$ etm.2017.4206

\begin{abstract}
The diagnostic criteria of osteoporosis were established based on bone mineral density (BMD). Therefore, BMD measured by dual energy X-ray absorptiometry has been recognized as the gold standard to diagnose osteoporosis. However, discrepancies between fracture risk and BMD have been recognized. Bone is composed of collagen scaffold reinforced by hydroxyapatite. Both protein scaffold and hydroxyapatite are involved in bone quality. BMD may indicate bone mineralization but potentially fail to assess the protein scaffold. Vitamin K contributes to bone mineralization and as a protein scaffold. A deficiency of vitamin $\mathrm{K}$ upregulates the level of serum undercarboxylated osteocalcin (ucOC), and serum ucOC correlates with fracture risk. However, direct association of ucOC and bone quality has not been demonstrated. For the present study, a total of 49 healthy young Japanese female college students underwent calcaneal; quantitative ultrasound sonometry (QUS) and determination of serological bone metabolic markers. QUS parameters were significantly correlated with serum 25-hydroxyvitamin D (25-OH-D) concentrations $(\mathrm{P}<0.05)$. A
\end{abstract}

Correspondence to: Dr Yoshio Suzuki, Graduate School of Health and Sports Science, Juntendo University, 1-1 Hiraga-gakuendai, Inzai, Chiba 270-1695, Japan

E-mail: yssuzuki@juntendo.ac.jp

Abbreviations: BMD, bone mineral density; DXA, dual energy X-ray absorptiometry; Gla, $\gamma$-carboxyglutamic residue; ucOC, undercarboxylated osteocalcin; 25-OH-D, 25-hydroxyvitamin D; QUS, quantitative ultrasound sonometry; BH, body height; BW, body weight; BMI, body mass index; SOS, speed of sound; TI, transmission index; OSI, osteo-sono-assessment index; $1,25-\mathrm{OH}_{2}-\mathrm{D}$, 1,25-dihydroxyvitamin D; RIA, radioimmunoassay; FGF-23, fibroblast growth factor-23; NTX, N-telopeptide of type I collagen; TRACP-5b, tartrate resistant acid phosphatase-5b; PTH, parathyroid hormone; BAP, bone alkaline phosphatase; $\mathrm{Ca}$, calcium; P, phosphorus; TSK, tsukushin; SXR, steroid and xenobiotic receptor; MATN2, matrilin-2

Key words: vitamin K-dependent bone protein, vitamin K, osteoporosis, clinical laboratory techniques, bone matrix significant negative correlation was also identified between log transformed serum ucOC concentrations [Ln(ucOC)] and a QUS parameter, speed of sound (SOS) $(\mathrm{P}<0.05)$. Stepwise multiple regression analysis indicated that $\mathrm{Ln}(\mathrm{ucOC})$ was an independent determinant of SOS, and 25-OH-D was an independent determinant of the other two QUS parameters, transmission index (TI) and synthetic parameter osteo-sono-assessment index. As vitamin $\mathrm{D}$ is involved in bone mineralization, TI may reflect the mineralization. Correlation of vitamin $\mathrm{K}$ status, indicated by ucOC, with SOS may clarify the correlation between vitamin K status and bone quality, although the material factors that connect them have not been identified.

\section{Introduction}

The diagnostic criteria of osteoporosis was established based on bone mineral density (BMD) by the World Health Organization in 1994 (1). Therefore, BMD measured by dual energy X-ray absorptiometry (DXA) has been recognized as the gold standard to diagnose osteoporosis. However, discrepancies between fracture risk and BMD have been recognized $(2,3)$.

Bone is composed of protein scaffolds adhered and reinforced by hydroxyapatite (4). Bone quality is affected by vitamin $\mathrm{D}$ and other nutrients including vitamin $\mathrm{K}$, calcium (Ca) and phosphorus (P). Vitamin D is involved in bone mineralization, maintaining the serum level of the major bone minerals, $\mathrm{Ca}$ and $\mathrm{P}$ (5). Vitamin $\mathrm{K}$ serves as a coenzyme for $\gamma$-carboxylation of glutamic residues in protein to produce the $\gamma$-carboxyglutamic (Gla) residue. Osteocalcin, the second most abundant protein in bone, is a Gla-containing protein (6). Therefore, the level of serum undercarboxylated osteocalcin (ucOC) is influenced by the status of vitamin K (7) and associated with low bone mass (8).

The association of vitamin $\mathrm{D}$ status, indicated by the concentration of serum 25-hydroxyvitamin D (25-OH-D) and BMD has been demonstrated (9). By contrast, no association between ucOC and BMD was reported, despite an association with fracture risk being observed $(2,3)$.

Quantitative ultrasound sonometry (QUS) has been widely used to assess bone quality (10-12). However, a meta-analysis concluded it does not correspond sufficiently to the DXA diagnosis (13). An association of QUS parameters with bone turnover 
markers has been reported in Caucasian postmenopausal elderly women (14), German men (15) and the Framingham Offspring cohort (16). Therefore, QUS may serve a role in assessing bone quality reflected by vitamin $\mathrm{K}$ status.

The present study aimed to assess the correlation between QUS parameters and bone metabolic parameters including serum ucOC in healthy young Japanese females.

\section{Patients and methods}

Patients. A total of 49 healthy female college students were recruited from a dormitory of Juntendo University (Chiba, Japan) in April 2013 and their food and drink intake was not controlled by the study. Any subjects that were undergoing treatment for pre-existing diseases or conditions were not included in the present study. Patient ages and anthropometric characters were as follows: Age, $18.5 \pm 0.9$ years; body height

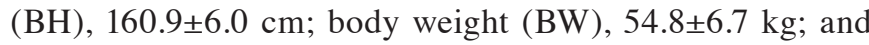
body mass index (BMI), $21.1 \pm 1.9 \mathrm{~kg} / \mathrm{m}^{2}$.

The present study was conducted according to the guidelines provided by the Declaration of Helsinki and all procedures involving human subjects were approved by the Ethics Committee of Juntendo University Graduate School of Health and Sports Science (Chiba, Japan; Approval no. 25-1). Written informed consent was obtained from all patients.

Bone quality. Calcaneal bone quality was assessed using the AOS-100NW ultrasound bone densitometry system (Hitachi, Ltd., Tokyo, Japan) according to the manufacturer's protocol. This quantitative ultrasonometry system measures two types of indices: The speed of sound (SOS) and the transmission index (TI). SOS is the ultrasound velocity that penetrates the calcaneus. Since this velocity depends on the density of the calcaneus, a higher SOS indicates a higher density. TI is an index related to the frequency-dependent attenuation of the ultrasound penetrating the heel. A higher TI indicates greater bone mass. A synthetic parameter, the osteo-sono-assessment index (OSI), is computed from SOS and TI according to the following equation: $\mathrm{OSI}=(\mathrm{SOS})^{2} \mathrm{x}$ TI $(17)$. It has been previously confirmed by DXA measurements that calcaneal OSI is associated with BMD in the distal radius (18).

Serological indices. Venous blood was collected from the cubital vein one morning in May 2013 prior to breakfast. According to the protocols provided by the manufacturers, serological indices were analyzed using the following reagents and apparatus: Serum 1,25-dihydroxyvitamin D (1,25- $\left.\mathrm{OH}_{2}-\mathrm{D}\right)$ and 25-OH-D were measured by radioimmunoassay (RIA) using $1,25(\mathrm{OH})_{2} \mathrm{D}$ RIA kits (Immunodiagnostic Systems Holdings PLC, Tyne and Wear, UK) and 25-hydroxyvitamin D ${ }^{125}$ I RIA Kits (DiaSorin S.p.A., Saluggia, Italy). Serum fibroblast growth factor-23 (FGF-23), N-telopeptide of type I collagen (NTX), and tartrate resistant acid phosphatase-5b (TRACP-5b) underwent ELISA using an FGF-23-ELISA kit (Kainos Laboratories, Inc., Tokyo, Japan), Osteomark ${ }^{\circledR}$ NTx serum ELISA (Alere, Inc., Waltham, MA, USA), and Osteolinks-TRACP-5b ${ }^{\circledR}$ test kit (DS Pharma Biomedical, Co., Ltd., Osaka, Japan) kits, respectively. Serum intact parathyroid hormone (PTH) and bone alkaline phosphatase (BAP) were analyzed using electrochemiluminescence immunoassay kits (Roche Diagnostics K.K., Tokyo, Japan;
Beckman Coulter, Brea, CA, USA, respectively). Serum Ca and P were measured using a 7170 chemistry analyzer (Hitachi, Ltd.). Serum ucOC was assayed using a Picolumi ${ }^{\circledR}$ ucOC kit (Eisai, Co., Ltd., Tokyo, Japan). Analysis of samples was completed by a Japanese clinical analytical laboratory (SRL, Inc., Tokyo, Japan) and the references ranges used are provided in Table I.

Statistical analysis. Descriptive data are presented as means \pm standard deviation, unless otherwise noted. The normality of the data was assessed by the Shapiro-Wilk test. Variables not normally distributed were natural log transformed.

Associations between two parameters were assessed using Pearson's correlation coefficient, with $r>0.2$ considered to indicate statistical significance. A stepwise multiple linear regression analysis was performed to explore determinants of QUS parameters, removing the variables if the probability of $\mathrm{F} \geq 0.1$. The following plausible anthropometric and serological parameters were included in the model: Age, BW, BH, BMI, 1,25- $\mathrm{OH}_{2}-\mathrm{D}, 25-\mathrm{OH}-\mathrm{D}, \mathrm{Ca}, \mathrm{P}, \mathrm{TRACP}-5 \mathrm{~b}, \mathrm{NTX}, \mathrm{PTH}$, and Log transformed concentrations of BAP, ucOC and FGF-23; denoted as $\mathrm{Ln}(\mathrm{BAP}), \mathrm{Ln}(\mathrm{ucOC})$ and $\mathrm{Ln}(\mathrm{FGF}-23)$, respectively.

$\mathrm{P}<0.05$ was considered to represent a statistically significant difference. The statistical analysis was performed using SPSS software, version 19 (IBM SPSS, Armonk, NY, USA).

\section{Results}

Serological markers. The mean 25-OH-D concentration $(23.1 \mathrm{ng} / \mathrm{ml})$ was above the vitamin D insufficiency borderline (20 ng/ml); however, 16 patients $(33 \%)$ did not exceed the borderline. By contrast, the mean $1,25-\mathrm{OH}_{2}-\mathrm{D}(64.77 \mathrm{pg} / \mathrm{ml})$ was higher than the reference range $(20.0-60.0 \mathrm{pg} / \mathrm{ml})$. The mean ucOC $(7.851 \mathrm{ng} / \mathrm{ml})$ was higher than the cut-off level $(<4.5 \mathrm{ng} / \mathrm{ml})$ and only 8 subjects $(16 \%)$ were below the cut-off level. The mean NTX [23.02 nmol bone collagen equivalent $(\mathrm{BCE}) / \mathrm{l}]$ and BAP $(14.78 \mathrm{ng} / \mathrm{ml})$ exceeded the reference ranges of NTX (11.5-37.5 nmol BCE/l) and BAP (6.4-28.3 ng/ml), and $7(14 \%)$ and $25(51 \%)$ subjects were within the ranges for NTX and BAP, respectively. Other parameters were primarily distributed within the reference range. Blood biomarker concentrations are summarized in Table I.

QUS and anthropometric parameters. The mean SOS $(1,600 \mathrm{~m} / \mathrm{sec}), \mathrm{TI}(1.223)$ and OSI $\left(3.140 \times 10^{6}\right)$ were higher than the means of reference values from matched age and gender Japanese individuals. Even the minimum values were within the mean $\pm 2 x$ standard deviations of all parameters (Table II). Reference values were provided by the ultrasonometry equipment manufacturer (Hitachi, Ltd.).

All QUS parameters were significantly associated with BW and BMI, and TI exhibited a significant association with $\mathrm{BH}$ (Table III). The strongest correlation was identified between TI and BW ( $\mathrm{r}=0.493)$. SOS was primarily associated with BMI $(\mathrm{r}=0.364)$. The synthetic variable OSI exhibited significant associations with BW ( $\mathrm{r}=0.455)$ and BMI ( $\mathrm{r}=0.410$; Table III).

Correlations between serological and QUS parameters. The association between serological and QUS parameters was assessed using the Pearson's correlation coefficient. The concentrations of BAP, ucOC and FGF-23 were natural log 
Table I. Serological parameters of the patients.

\begin{tabular}{lccc}
\hline Variable & Reference (range) & Mean \pm SD & Range \\
\hline $1,25-\mathrm{OH} \mathrm{H}_{2} \mathrm{D}(\mathrm{pg} / \mathrm{ml})$ & $20.0-60.0^{\mathrm{a}}$ & $64.8 \pm 15.6$ & $35.4-99.2$ \\
$25-\mathrm{OH}-\mathrm{D}(\mathrm{ng} / \mathrm{ml})$ & $7-41^{\mathrm{b}}$ & $23 \pm 5$ & $13-34$ \\
$\mathrm{Ca}(\mathrm{mg} / \mathrm{dl})$ & $8.2-10.2^{\mathrm{c}}$ & $9.4 \pm 0.3$ & $8.8-9.9$ \\
$\mathrm{P}(\mathrm{mg} / \mathrm{dl})$ & $2.4-4.3^{\mathrm{d}}$ & $4.2 \pm 0.4$ & $3.4-4.9$ \\
$\mathrm{TRACP}-5 \mathrm{~b}(\mathrm{mU} / \mathrm{dl})$ & $120-420^{\mathrm{e}}$ & $268 \pm 65$ & $159-437$ \\
$\mathrm{NTX}(\mathrm{nmol} \mathrm{BCE} / \mathrm{l})$ & $7.5-16.5^{\mathrm{e}}$ & $23.0 \pm 5.9$ & $11.5-37.5$ \\
$\mathrm{BAP}(\mathrm{ng} / \mathrm{ml})$ & $2.9-14.5^{\mathrm{e}}$ & $14.8 \pm 5.0$ & $6.4-28.3$ \\
PTH $(\mathrm{pg} / \mathrm{ml})$ & $10-65^{\mathrm{a}}$ & $37 \pm 14$ & $15-71$ \\
$\mathrm{ucOC}(\mathrm{ng} / \mathrm{ml})$ & $<4.5^{\mathrm{e}}$ & $7.85 \pm 4.48$ & $1.49-24.9$ \\
FGF-23 $(\mathrm{pg} / \mathrm{ml})$ & - & $43 \pm 10$ & $25-78$
\end{tabular}

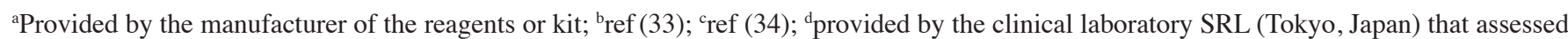
subjects; ${ }^{e}$ ref (32). SD, standard deviation; 1 ,25- $\mathrm{OH}_{2}-\mathrm{D}, 1$, 25-dihydroxyvitamin D; 25-OH-D, 25-hydroxyvitamin D; Ca, calcium; P, phosphorus; TRACP-5b, tartrate resistant acid phosphatase-5b; NTX, N-telopeptide of type I collagen; BAP, bone alkaline phosphatase; PTH, parathyroid hormone; ucOC, undercarboxylated osteocalcin; FGF-23, fibroblast growth factor-23.

Table II. QUS parameters measured in subjects.

\begin{tabular}{llcc}
\hline Variable & $\begin{array}{c}\text { Reference } \\
(\text { mean } \pm \text { SD) }\end{array}$ & $\begin{array}{c}\text { Reference } \\
(\text { range })\end{array}$ & Mean \pm SD \\
\hline SOS $(\mathrm{m} / \mathrm{sec})$ & $1.600 \pm 28$ & $1.539-1,651$ & $1.564 .4 \pm 21.7$ \\
TI & $1.223 \pm 0.107$ & $1.005-1.478$ & $1.091 \pm 0.074$ \\
OSI, x $10^{6}$ & $3.140 \pm 0.370$ & $2.417-4.032$ & $2.698 \pm 0.298$
\end{tabular}

Reference range indicates the distribution in healthy Japanese females provided by the clinical laboratory. SD, standard deviation; QUS, quantitative ultrasound sonometry; SOS, speed of sound; TI, transmission index; OSI, osteo-sono-assessment index.

Table III. Associations between QUS parameters with age, body height, body weight and body mass index of subjects analyzed by Pearson's correlation coefficient.

\begin{tabular}{lccccc}
\hline & & Age & $\begin{array}{c}\text { Body } \\
\text { height }\end{array}$ & $\begin{array}{c}\text { Body } \\
\text { weight }\end{array}$ & $\begin{array}{c}\text { Body mass } \\
\text { index }\end{array}$ \\
\hline SOS & $\mathrm{r}$ & 0.155 & 0.046 & $0.301^{\mathrm{a}}$ & $0.364^{\mathrm{b}}$ \\
& P-value & 0.289 & 0.756 & 0.036 & 0.010 \\
TI & $\mathrm{r}$ & 0.012 & $0.295^{\mathrm{a}}$ & $0.493^{\mathrm{b}}$ & $0.408^{\mathrm{b}}$ \\
& P-value & 0.933 & 0.039 & $<0.001$ & 0.004 \\
OSI & $\mathrm{r}$ & 0.059 & 0.233 & $0.455^{\mathrm{b}}$ & $0.410^{\mathrm{b}}$ \\
& P-value & 0.687 & 0.107 & 0.001 & 0.003 \\
\hline
\end{tabular}

${ }^{\mathrm{a}} \mathrm{P}<0.05 ;{ }^{\mathrm{b}} \mathrm{P}<0.01$. QUS, quantitative ultrasound sonometry; SOS, speed of sound; $r$, Pearson's correlation coefficient; TI, transmission index; OSI, osteo-sono-assessment index.

transformed to achieve a normal distribution (Table I). The level of 25-OH-D indicated a significant positive correlation
$(\mathrm{P}<0.05)$ with all QUS parameters: $\mathrm{SOS}(\mathrm{r}=0.293), \mathrm{TI}(\mathrm{r}=0.315)$ and OSI (r=0.324). Ln(ucOC) correlated negatively with SOS $(r=-0.298$; Fig. 1). The other serological parameters did not exhibit any significant correlations with QUS parameters (Table IV).

Stepwise regression analysis to examine determinants of QUS parameters. From the correlation analysis, anthropometric parameters, the level of 25-OH-D and $\mathrm{Ln}$ (ucOC) were considered to be associated with QUS parameters, although the correlation coefficients were weak $(0.2<\mathrm{r}<0.4$; Table IV). However, the correlation with anthropometric parameters may conceal the associations between serological and QUS parameters. Therefore, stepwise multiple linear regression analysis was performed to examine the determinants for QUS parameters. In the model, in addition to anthropometric parameters, 25-OH-D and $\mathrm{Ln}(\mathrm{ucOC})$, the following parameters were included: Bone formation marker $\mathrm{Ln}(\mathrm{BAP})$, bone absorption markers (NTX and TRAP-5b), and markers reflecting $\mathrm{Ca}$ and $\mathrm{P}$ status [Ca, P, 1,25- $\mathrm{OH}_{2}-\mathrm{D}, \mathrm{PTH}$ and $\left.\mathrm{Ln}(\mathrm{FGF}-23)\right]$.

The regression analysis provided well-fitted predictive equations $(\mathrm{P}<0.003)$. This indicated that $\mathrm{Ln}(\mathrm{ucOC})$ and BMI were independent determinants of SOS, whereas 25-OH-D and BW were independent determinants of TI and OSI. For OSI, Ln(ucOC) was also selected in the final model, but the probability was not significant $(\mathrm{P}=0.100$; Table V).

\section{Discussion}

The present study examined bone metabolic markers and QUS parameters in Japanese females. Overall, 33\% of the subjects exhibited vitamin D deficiency (25-H-D $<20 \mathrm{ng} / \mathrm{ml})$ and $84 \%$ were suspected to have vitamin $\mathrm{K}$ insufficiency according to their levels of ucOC $(>4.5 \mathrm{ng} / \mathrm{ml})$. The bone quality was assessed by QUS, and typically was above the reference range of an ageand gender-matched Japanese population. All QUS parameters indicated positive correlations with BW and BMI. Significant but moderately weak correlations were also identified for SOS 
Table IV. Correlations between QUS parameters and serological parameters.

\begin{tabular}{lcrlllllllll}
\hline & & $1,25-O H-D$ & $25-O H-D$ & $\mathrm{Ca}$ & $\mathrm{P}$ & TRACP-5b & NTX & Ln(BAP) & PTH & Ln(ucOC) & Ln(FGF-23) \\
\hline \multirow{2}{*}{ SOS } & $\mathrm{r}$ & -0.110 & $0.293^{\mathrm{a}}$ & 0.033 & -0.019 & -0.068 & -0.242 & -0.089 & -0.109 & $-0.298^{\mathrm{a}}$ & 0.198 \\
& P-value & 0.454 & 0.041 & 0.819 & 0.899 & 0.641 & 0.094 & 0.544 & 0.455 & 0.038 & 0.173 \\
TI & $\mathrm{r}$ & -0.007 & $0.315^{\mathrm{a}}$ & 0.158 & -0.047 & 0.125 & -0.105 & 0.087 & -0.043 & -0.216 & 0.109 \\
& P-value & 0.960 & 0.027 & 0.278 & 0.748 & 0.390 & 0.472 & 0.554 & 0.767 & 0.137 & 0.455 \\
OSI & $\mathrm{r}$ & -0.041 & $0.324^{\mathrm{a}}$ & 0.129 & -0.047 & 0.069 & -0.145 & 0.034 & -0.060 & -0.253 & 0.141 \\
& P-value & 0.779 & 0.023 & 0.379 & 0.750 & 0.636 & 0.320 & 0.817 & 0.681 & 0.080 & 0.334 \\
\hline
\end{tabular}

${ }^{\mathrm{a}} \mathrm{P}<0.05$. r, Pearson's correlation coefficient; QUS, quantitative ultrasound sonometry; SOS, speed of sound; TI, transmission index; OSI, osteo-sono-assessment index; 1,25-OH-D, 1,25-hydroxyvitamin D; 25-OH-D, 25-hydroxyvitamin D; Ca, calcium; P, phosphorus; TRACP-5b, tartrate resistant acid phosphatase-5b; NTX, N-telopeptide of type I collagen; Ln(BAP), log transformed serum concentration of bone alkaline phosphatase; PTH, parathyroid hormone; Ln(ucOC), log transformed serum concentration of undercarboxylated osteocalcin; Ln(FGF-23), long transformed serum concentration of fibroblast growth factor- 23 .

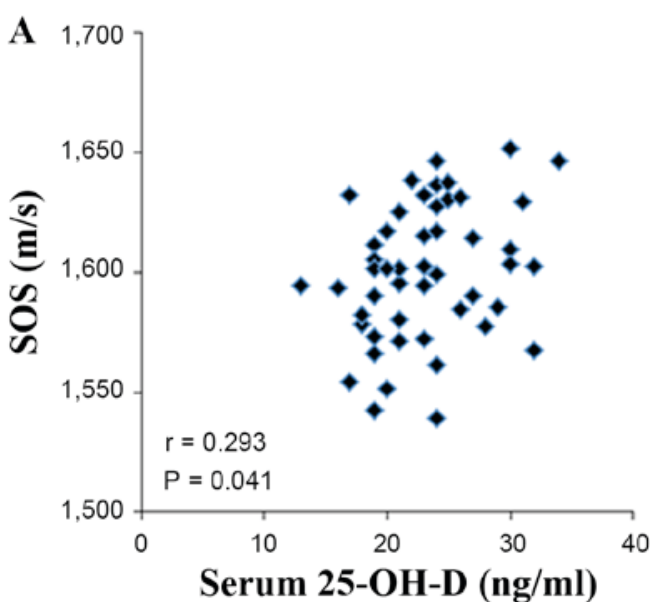

C

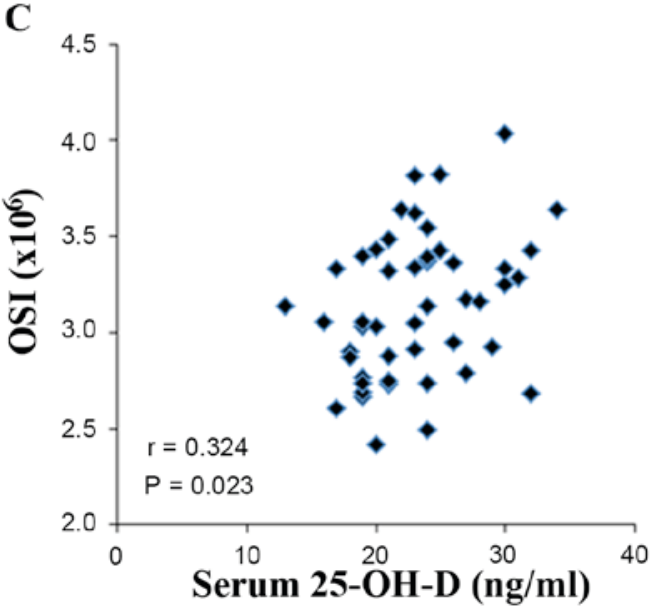

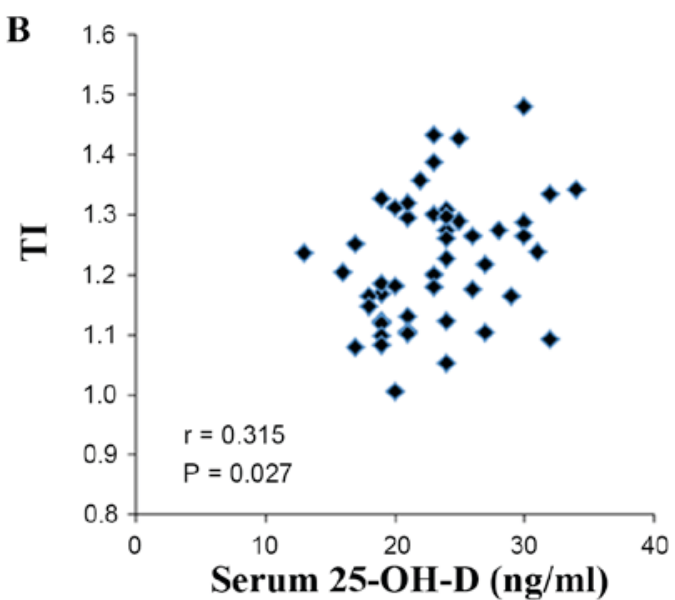

D

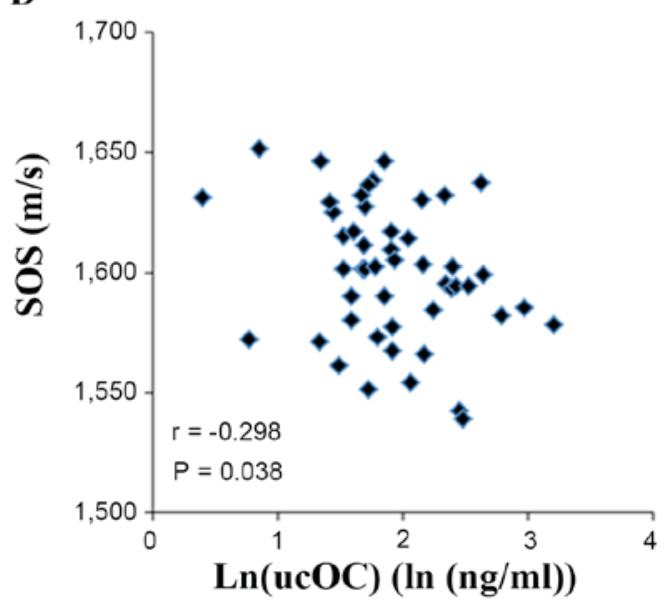

Figure 1. Correlations between serological markers and QUS parameters. (A) Serum 25-OH-D and SOS, (B) serum 25-OH-D and SOS, (C) serum 25-OH-D and (D) Log transformed serum ucOC and SOS. P<0.05 represents a statistically significant difference. 25-OH-D, 25-hydroxyvitamin D; SOS, speed of sound; ucOC, undercarboxylated osteocalcin.

with ucOC, and for TI and OSI with 25-OH-D. Stepwise multiple regression analysis demonstrated that $\mathrm{Ln}(\mathrm{ucOC})$ and BMI were independent determinants of SOS, while 25-OH-D and BW were independent determinants of TI and OSI.

Serum 25-OH-D correlated with TI and OSI, but not with SOS. Sohl et al (19) reported that 25-OH-D was associated with BMD and another QUS parameter broadband ultrasound attenuation (BUA), but not with SOS, in elderly Dutch subjects with a low-to-normal BMI $\left(<25 \mathrm{~kg} / \mathrm{m}^{2}\right)$. Tanabe et al (20) demonstrated the effect of genetic variation. A significant correlation between QUS parameters and serum 25-OH-D was observed in FF type but not in ff type vitamin D receptor 
Table V. Stepwise regression analysis to explore the determinants for QUS parameters.

\begin{tabular}{|c|c|c|c|c|}
\hline Dependent variable & Independent variable & Std coefficient & $\begin{array}{c}\text { P-values } \\
\text { (Coefficient) }\end{array}$ & $\begin{array}{c}\text { P-values } \\
\text { (Model) }\end{array}$ \\
\hline \multirow[t]{2}{*}{ SOS } & BMI & 0.363 & $0.008^{\mathrm{b}}$ & \multirow[t]{2}{*}{$0.003^{\mathrm{b}}$} \\
\hline & $\operatorname{Ln}(\mathrm{ucOC})$ & -0.297 & $0.027^{\mathrm{a}}$ & \\
\hline \multirow[t]{2}{*}{ TI } & $\mathrm{BW}$ & 0.488 & $<0.001^{\mathrm{b}}$ & \multirow[t]{2}{*}{$<0.001^{\mathrm{b}}$} \\
\hline & $25-\mathrm{OH}-\mathrm{D}$ & 0.308 & $0.014^{\mathrm{a}}$ & \\
\hline \multirow[t]{3}{*}{ OSI } & $\mathrm{BW}$ & 0.457 & $<0.001^{\mathrm{b}}$ & \multirow[t]{3}{*}{$<0.001^{\mathrm{b}}$} \\
\hline & 25-OH-D & 0.272 & $0.033^{\mathrm{a}}$ & \\
\hline & $\mathrm{Ln}(\mathrm{ucOC})$ & -0.207 & 0.100 & \\
\hline
\end{tabular}

${ }^{\mathrm{a}} \mathrm{P}<0.05$; ${ }^{\mathrm{P}}<0.01$. Std coefficient, standardized coefficient; $\mathrm{P}$ (Coefficient), P-value of the coefficient; $\mathrm{P}$ (Model), P-value of the model; CI, confidence index; SOS, speed of sound; TI, transmission index; OSI, osteo-sono-assessment index; BMI, body mass index; Ln(ucOC), log transformed serum concentration of undercarboxylated osteocalcin; BW, body weight; 25-OH-D, 25-hydroxyvitamin D.

polymorphism in young Japanese adults. Since the incidence rate of type was small (12\%) in the Japanese population (20), these correlations may be observed. However, the polymorphism was not assessed in the present subjects.

Bone is composed of collagen scaffold strengthened by calcium hydroxyapatite (4). Since serum 25-OH-D indicates vitamin $\mathrm{D}$ status, it affects bone mineralization via serum calcium concentration. Therefore, the association of 25-OH-D and BMD was demonstrated in subjects of the US National Health and Nutrition Examination Surveys (9). The association of 25-OH-D and TI and OSI was thus considered to be indicative of BMD.

On stepwise multiple regression analysis, $\mathrm{Ln}(\mathrm{ucOC})$ and BMI were demonstrated to be independent determinants of SOS, and 25-OH-D and BW were independent determinants of TI and OSI. The associations of anthropometric parameters with QUS parameters were generally in accordance with Brunner et al (11). The major finding of the present study was the clarification of the independent association between ucOC and SOS.

An association between serum ucOC and hip fracture has been reported $(8,21,22)$. Tsugawa et al $(2)$ reported the negative association of vertebrate fracture incident with the serum phylloquinone (vitamin $\mathrm{K}_{1}$ ) level. Stratified by the serum vitamin $\mathrm{K}_{1}$ level, vertebral fracture incidents were higher in the low vitamin $\mathrm{K}_{1}$ group; however, no significant difference was observed in BMD (2). Specifically, it was observed that the correlation coefficient between log transformed serum ucOC and fracture incidents was positive, though not significant $[\mathrm{P}=0.088 ;(2)]$. Shiraki et al (3) also documented that fracture risk was associated with serum ucOC but not with BMD in osteoporotic patients. A meta-analysis indicated that vitamin $\mathrm{K}$ antagonists increased the fracture risk, but did not affect BMD (23). Therefore, vitamin K status should be associated with fracture risk, independent of BMD.

Vitamin K contributes to bone quality via two mechanisms. First, it serves as a coenzyme to mature Gla proteins. Bone Gla proteins hold calcium ions to strengthen the bone. This mechanism may contribute to bone mineralization (24). Second, it serves as a ligand for the steroid and xenobiotic receptor (SXR), to stimulate the expression of 'tsukushin' (TSK) (25).
TSK is an extracellular protein involved in collagen assembly. SXR also stimulates matrilin-2 (MATN2), a matrix protein participating in the formation of fibrillar or filamentous structures. TSK and MATN2 are associated with collagens to promote collagen-accumulation in osteoblastic cells (26). This mechanism may affect the scaffold structure of bone.

The bone hydroxyapatite accounts for $60 \%$ of human bone, and its increase strengthens but decreases the flexibility of the bone. The cross-linking of the collagen scaffold also affects bone strength (4). Therefore, mineralization and scaffold structure contribute to bone quality. Bone mineralization is measured by DXA, as BMD, but BMD may not represent quality of the scaffold structure. This may account for the absence of an association of BMD with fracture risk $(2,3)$.

The association of SOS with BMD has been controversial: Hans et al (12) supported the association, but Brooke-Wavell et al (10) did not. The results of the present study clarify the association of SOS with ucOC in young Japanese females, at the age where the highest BMD is identified, independent of anthropometric and other serological bone markers.

According to the QUS parameters, the subjects in the current study had an improved bone quality than the same age/sex population of Japanese individuals. All subjects were physically active college students, as the majority of them belonged to a college sports club, though they were not initially selected based on physical activity. This may account for the improved bone quality of this group, since physical activity positively correlates with bone status $(27,28)$.

The vitamin D status of the subjects was also improved, compared with the same age/sex population of Japanese individuals $(29,30)$. Nakamura et al (29) indicated that the level of 25-OH-D in Japanese females aged 19-24 years were $13.7 \pm 4.8 \mathrm{ng} / \mathrm{ml}$. Ohta et al (30) reported that the $25-\mathrm{OH}-\mathrm{D}$ level in 274 Japanese women aged $19-25$ years was $18.7 \pm 4.8 \mathrm{ng} / \mathrm{ml}$. As with bone quality, the physical activity of the subjects may contribute to the vitamin D status $(27,28)$, though it is unknown whether subjects in the Ohta et al study were more physically active.

The mean NTX and BAP values were higher than the reference ranges. Both parameters are indicators of bone 
turnover, which is high in childhood and decreases with age; they remain high at the age of the current subjects' (31). By contrast, the reference ranges were established in adult women aged 30-44 years (32), which may explain the discrepancy.

The correlation of QUS parameters with anthropometric parameters was in accordance with a previous study by Brunner et al (11), where positive correlations were identified between SOS, BMI and BW in elderly German women using an Achilles + Solo ultrasound bone densitometer (GE Lunar Corporation, Madison, WI, USA). Correlations were also identified in BUA and the synthetic parameter stiffness index calculated from SOS and BUA.

The limitations in this study were as follows: The major limitation was the absence of DXA assessment. The discrepancy of BMD with SOS and ucOC should be assessed in the same population at the same time. Second, the study subjects were a specific population, having almost the same ages and the sample size was small. Although the majority of the results were in accordance with previous studies, further study on a variety of subjects should be warranted to generalize the results. Third, bone quality is influenced by ethnicity, sex, age, nutritional status, physical activity, sun exposure and genetic background. In the current study, ethnicity, sex and age were identical; Ca, P, vitamin D and vitamin $\mathrm{K}$ were assessed by serological indices, while physical activity, sun exposure, and genetic polymorphism were not assessed. These factors should be monitored and controlled in a future study. Finally, although correlation of serum ucOC with SOS was demonstrated, serum ucOC is biological and SOS is physical, and material factors to connect them were not identified. Therefore, further studies are warranted.

In conclusion, serum 25-OH-D and ucOC were identified to have an association with calcaneal QUA parameters, TI and SOS, respectively, independent from other bone turnover indices and anthropometric parameters. As vitamin D is involved in bone mineralization, TI may reflect the level of mineralization. Vitamin $\mathrm{K}$ status, indicated by ucOC, was demonstrated to correlate with SOS. This may facilitate future studies to clarify the presence of a correlation between vitamin $\mathrm{K}$ status and bone quality, although the factors that connect them have not yet been identified.

\section{Acknowledgements}

The authors of the present study would like to thank all subjects who participated in the study. A part of this study was presented at a local meeting of the Japanese Society of Physical Fitness and Sports Medicine in March 2014 in Tokyo, Japan. The present study was funded in part by the Japan Dairy Association (J-milk; Tokyo, Japan) and the Ministry of Education, Culture, Sports, Science and Technology (MEXT) - Supported Program for the Strategic Research Foundation at Private Universities (grant no. S1101008; Tokyo, Japan).

\section{References}

1. Assessment of fracture risk and its application to screening for postmenopausal osteoporosis. Report of a WHO study group. World Health Organ Tech Rep Ser 843: 1-129, 1994.

2. Tsugawa N, Shiraki M, Suhara Y, Kamao M, Ozaki R, Tanaka K and Okano T: Low plasma phylloquinone concentration is associated with high incidence of vertebral fracture in Japanese women. J Bone Miner Metab 26: 79-85, 2008.
3. Shiraki M, Yamazaki Y, Shiraki Y, Hosoi T, Tsugawa N and Okano T: High level of serum undercarboxylated osteocalcin in patients with incident fractures during bisphosphonate treatment. J Bone Miner Metab 28: 578-584, 2010.

4. Seeman E and Delmas PD: Bone quality-the material and structural basis of bone strength. N Engl J Med 354: 2250-2261, 2006.

5. Carmeliet G, Dermauw V and Bouillon R: Vitamin D signaling in calcium and bone homeostasis: A delicate balance. Best Pract Res Clin Endocrinol Metab 29: 621-631, 2015.

6. Cashman KD: Diet, Nutrition, and bone health. J Nutr 137 (11 Suppl): 2507S-2512S, 2007.

7. Sokoll LJ, Booth SL, O'Brien ME, Davidson KW, Tsaioun KI and Sadowski JA: Changes in serum osteocalcin, plasma phylloquinone, and urinary gamma-carboxyglutamic acid in response to altered intakes of dietary phylloquinone in human subjects. Am J Clin Nutr 65: 779-784, 1997.

8. Vergnaud P, Garnero P, Meunier PJ, Bréart G, Kamihagi K and Delmas PD: Undercarboxylated osteocalcin measured with a specific immunoassay predicts hip fracture in elderly women: The EPIDOS study. J Clin Endocrinol Metab 82: 719-724, 1997.

9. Gutiérrez OM, Farwell WR, Kermah D and Taylor EN: Racial differences in the relationship between vitamin $\mathrm{D}$, bone mineral density, and parathyroid hormone in the National health and nutrition examination survey. Osteoporos Int 22: 1745-1753, 2011.

10. Brooke-Wavell K, Khan AS, Taylor R and Masud T: Lower calcaneal bone mineral density and broadband ultrasonic attenuation, but not speed of sound, in South Asian than white European women. Ann Hum Biol 35: 386-393, 2008.

11. Brunner C, Pons-Kühnemann J and Neuhäuser-Berthold $\mathbf{M}$ : Impact of age, anthropometric data and body composition on calcaneal bone characteristics, as measured by quantitative ultrasound (QUS) in an older German population. Ultrasound Med Biol 37: 1984-1992, 2011.

12. Hans D, Wu C, Njeh CF, Zhao S, Augat P, Newitt D, Link T, Lu Y, Majumdar S and Genant HK: Ultrasound velocity of trabecular cubes reflects mainly bone density and elasticity. Calcif Tissue Int 64: 18-23, 1999.

13. Nayak S, Olkin I, Liu H, Grabe M, Gould MK, Allen IE, Owens DK and Bravata DM: Meta-analysis: Accuracy of quantitative ultrasound for identifying patients with osteoporosis. Ann Intern Med 144: 832-841, 2006.

14. Lappa V, Dontas IA, Trovas G, Constantelou E, Galanos A and Lyritis GP: Quantitative ultrasound is better correlated with bone mineral density and biochemical bone markers in elderly women. Clin Rheumatol 26: 1067-1073, 2007.

15. Kyvernitakis I, Saeger U, Ziller V, Bauer T, Seker-Pektas B and Hadji P: The effect of age, sex hormones, and bone turnover markers on calcaneal quantitative ultrasonometry in healthy German men. J Clin Densitom 16: 320-328, 2013.

16. McLean RR, Booth SL, Kiel DP, Broe KE, Gagnon DR, Tucker KL, Cupples LA and Hannan MT: Association of dietary and biochemical measures of vitamin $\mathrm{K}$ with quantitative ultrasound of the heel in men and women. Osteoporos Int 17: 600-607, 2006

17. Tsuda-Futami E, Hans D, Njeh CF, Fuerst T, Fan B, Li J, He YQ and Genant HK: An evaluation of a new gel-coupled ultrasound device for the quantitative assessment of bone. Br J Radiol 72: 691-700, 1999.

18. Sasaki M, Harata S, Kumazawa Y, Mita R, Kida K and Tsuge M: Bone mineral density and osteo sono assessment index in adolescents. J Orthop Sci 5: 185-191, 2000.

19. Sohl E, de Jongh RT, Swart KM, Enneman AW, van Wijngaarden JP, van Dijk SC, Ham AC, van der Zwaluw NL, Brouwer-Brolsma EM, van der Velde N, et al: The association between vitamin D status and parameters for bone density and quality is modified by body mass index. Calcif Tissue Int 96: 113-122, 2015.

20. Tanabe R, Kawamura Y, Tsugawa N, Haraikawa M, Sogabe N, Okano T, Hosoi T and Goseki-Sone M: Effects of Fok-I polymorphism in vitamin D receptor gene on serum 25-hydroxyvitamin D, bone-specific alkaline phosphatase and calcaneal quantitative ultrasound parameters in young adults. Asia Pac J Clin Nutr 24: 329-335, 2015.

21. Szulc P, Arlot M, Chapuy MC, Duboeuf F, Meunier PJ and Delmas PD: Serum undercarboxylated osteocalcin correlates with hip bone mineral density in elderly women. J Bone Miner Res 9: 1591-1595, 1994.

22. Szulc P, Chapuy MC, Meunier PJ and Delmas PD: Serum undercarboxylated osteocalcin is a marker of the risk of hip fracture in elderly women. J Clin Invest 91: 1769-1774, 1993. 
23. Veronese N, Bano G, Bertozzo G, Granziera S, Solmi M, Manzato E, Sergi G, Cohen AT and Correll CU: Vitamin k antagonists' use and fracture risk: Results from a systematic review and meta-analysis. J Thromb Haemost 13: 1665-1675, 2015.

24. Bügel S: Vitamin $\mathrm{K}$ and bone health in adult humans. Vitam Horm 78: 393-416, 2008

25. Ichikawa T, Horie-Inoue K, Ikeda K, Blumberg B and Inoue S: Steroid and xenobiotic receptor SXR mediates vitamin K2-activated transcription of extracellular matrix-related genes and collagen accumulation in osteoblastic cells. J Biol Chem 281: 16927-16934, 2006.

26. Horie-Inoue K and Inoue S: Steroid and xenobiotic receptor mediates a novel vitamin K2 signaling pathway in osteoblastic cells. J Bone Miner Metab 26: 9-12, 2008.

27. Courteix D, Lespessailles E, Peres SL, Obert P, Germain P and Benhamou CL: Effect of physical training on bone mineral density in prepubertal girls: A comparative study between impact-loading and non-impact-loading sports. Osteoporos Int 8 : $152-158,1998$

28. Creighton DL, Morgan AL, Boardley D and Brolinson PG: Weight-bearing exercise and markers of bone turnover in female athletes. J Appl Physiol 90: 565-570, 2001.

29. Nakamura K, Nashimoto M, Tsuchiya Y, Obata A, Miyanishi K and Yamamoto M: Vitamin D insufficiency in Japanese female college students: A preliminary report. Int J Vitam Nutr Res 71: 302-335, 2001
30. Ohta H, Kuroda T, Onoe Y, Orito S, Ohara M, Kume M, Harada A, Tsugawa N, Okano T and Sasaki S: The impact of lifestyle factors on serum 25-hydroxyvitamin D levels: A cross-sectional study in Japanese women aged 19-25 years. J Bone Miner Metab 27: 682-688, 2009.

31. Orito S, Kuroda T, Onoe Y, Sato Y and Ohta H: Age-related distribution of bone and skeletal parameters in 1,322 Japanese young women. J Bone Miner Metab 27: 698-704, 2009.

32. Nishizawa Y, Ohta H, Miura M, Inaba M, Ichimura S, Shiraki M, Takada J, Chaki O, Hagino H, Fujiwara S, et al: Guidelines for the use of bone metabolic markers in the diagnosis and treatment of osteoporosis (2012 edition). J Bone Miner Metab 31: 1-15, 2013.

33. Kobayashi T, Okano T, Shida S, Okada K, Suginohara T, Nakao H, Kuroda E, Kodama $S$ and Matsuo T: Variation of 25-hydroxyvitamin-D3 and 25-hydroxy-vitamin-D2 levels in human-plasma obtained from 758 Japanese healthy-subjects. J Nutr Sci Vitaminol (Tokyo) 29: 271-281, 1983.

34. Takei I, Oguchi S, Ishibashi M, Ishida K, Sekiguchi H, Kikuchi H, Watanabe K, Sasaki A, Imamura C, Kohka K, Gonaikawa S, Aoki Y, Kano S, Kobayashi T and Miyazaki N: Reference ranges of endocrine, lipid and chemical laboratory tests at Keio University affiliated hospitals. Keio Igaku 80: 105-110, 2003. 